\title{
88.
}

\section{ON THE TRANSFORMATION OF AN ELLIPTIC INTEGRAL.}

[From the Cambridge and Dublin Mathematical Journal, vol. v. (1850), pp. 204-206.]

THE following is a demonstration of a formula proved incidentally by $\mathrm{Mr}$ Boole (Journal, vol. II. [1847] p. 7), in a paper "On the Attraction of a Solid of Revolution on an External Point."

Let

$$
U=\int_{-1}^{1} \frac{d x}{\sqrt{ }\left[\left(1-x^{2}\right)\left\{1-(m x+n)^{2}\right\}\right.} ;
$$

then, assuming

$$
i x=\frac{\alpha+i y}{1-i \alpha y}
$$

(so that $x= \pm 1$ gives $y= \pm 1$ ), we obtain

$$
\begin{gathered}
1-x^{2}=\frac{\left(1+\alpha^{2}\right)\left(1-y^{2}\right)}{(1-i \alpha y)^{2}}, \\
m x+n=\frac{(n-i m \alpha)+(m-i n \alpha) y}{1-i \alpha y} .
\end{gathered}
$$

Assume therefore

$$
i \alpha+(n-i m \alpha)(m-i n \alpha)=0,
$$

whence

$$
-i \alpha=\frac{\left(1-m^{2}-n^{2}\right)+\Delta}{2 m n} \quad\left(\Delta^{2}=1+m^{4}+n^{4}-2 m^{2}-2 n^{2}-2 m^{2} n^{2}\right)
$$


we find

$$
1-(m x+n)^{2}=\frac{1-(n-i m \alpha)^{2}}{(1-i \alpha y)^{2}}\left\{1-(m-i n \alpha)^{2} y^{2}\right\}
$$

and also

$$
d x=\frac{\left(1+\alpha^{2}\right) d y}{(1-i \alpha y)^{2}}
$$

whence

$$
U=\sqrt{\left\{\frac{1+\alpha^{2}}{1-(n-i m \alpha)^{2}}\right\}} \int_{-1}^{1} \frac{d y}{\sqrt{\left[\left(1-y^{2}\right)\left\{1-(m-i n \alpha)^{2} y^{2}\right\}\right]}},
$$

that is

$$
U=2 \sqrt{\left\{\frac{1+\alpha^{2}}{1-(n-i m \alpha)^{2}}\right)} \int_{0}^{1} \frac{d y}{\sqrt{\left[\left(1-y^{2}\right)\left\{1-(m-i n \alpha)^{2} y^{2}\right\}\right]}} .
$$

But since

$$
\begin{aligned}
& n-i m \alpha=\frac{1-m^{2}+n^{2}+\Delta}{2 n}, \\
& m-i n \alpha=\frac{1+m^{2}-n^{2}+\Delta}{2 m}
\end{aligned}
$$

we have

$$
\begin{aligned}
1-(n-i m \alpha)^{2} & =-\frac{\Delta}{2 n^{2}} \quad\left(\Delta+1-m^{2}+n^{2}\right), \\
1+\alpha^{2} & =-\frac{\Delta}{2 m^{2} n^{2}}\left(\Delta+1-m^{2}-n^{2}\right)
\end{aligned}
$$

and therefore

$$
\begin{aligned}
\frac{1+\alpha^{2}}{1-(n-i m \alpha)^{2}} & =\frac{1}{m^{2}} \frac{\Delta+1-m^{2}-n^{2}}{\Delta+1-m^{2}+n^{2}} \\
& =\frac{1}{m^{2}} \frac{\left(1-m^{2}-n^{2}+\Delta\right)\left(1-m^{2}+n^{2}-\Delta\right)}{\left(1-m^{2}+n^{2}+\Delta\right)\left(1-m^{2}+n^{2}-\Delta\right)}=\frac{2\left(1+m^{2}-n^{2}+\Delta\right)}{4 m^{2}}
\end{aligned}
$$

consequently

$$
U=\frac{1}{m} \sqrt{ }\left\{2\left(1+m^{2}-n^{2}+\Delta\right)\right\} \int_{0}^{1} \frac{d y}{\sqrt{\left[\left(1-y^{2}\right)\left\{1-\left(\frac{1+m^{2}-n^{2}+\Delta}{2 m}\right)^{2} y^{2}\right\}\right]}}
$$

Write

$$
k=\frac{1+m^{2}-n^{2}+\Delta}{2 m}, \quad \lambda^{2}=\frac{4 m}{(1+m)^{2}-n^{2}}
$$

then

$$
U=\frac{4 \sqrt{ } k}{\lambda} \frac{1}{\sqrt{\left\{(1+m)^{2}-n^{2}\right\}}} \int_{0}^{1} \frac{d y}{\sqrt{\left\{\left(1-y^{2}\right)\left(1-k^{2} y^{2}\right)\right\}}}
$$

where $\lambda$ and $k$ are connected by the relation that exists for the transformation of the second order, viz.

$$
\lambda=\frac{2 \sqrt{ } k}{1+k}
$$


as may be immediately verified; hence, assuming

$$
y=\frac{\lambda z}{\sqrt{ } k} \sqrt{\left(\frac{1-z^{2}}{1-\lambda^{2} z^{2}}\right)}
$$

which gives

$$
\int_{0}^{1} \frac{d y}{\sqrt{\left\{\left(1-y^{2}\right)\left(1-k^{2} y^{2}\right)\right\}}}=\frac{\lambda}{\sqrt{k}} \int_{0}^{1} \frac{d z}{\sqrt{\left\{\left(1-z^{2}\right)\left(1-\lambda^{2} z^{2}\right)\right\}}}
$$

we find

$$
U=\frac{4}{\sqrt{ }\left\{(1+m)^{2}-n^{2}\right\}} \int_{0}^{1} \frac{d z}{\sqrt{\left\{\left(1-z^{2}\right)\left(1-\frac{4 m}{(1+m)^{2}-n^{2}} z^{2}\right)\right\}}}
$$

that is

$$
\int_{-1}^{1} \frac{d x}{\sqrt{\left[\left(1-x^{2}\right)\{1-(m x+n)\}^{2}\right]}}=4 \int_{0}^{1} \frac{d z}{\sqrt{\left(1-z^{2}\right)\left[\left\{(1+m)^{2}-n^{2}\right\}-4 m z^{2}\right]}}
$$

Writing here

$$
x=\cos \theta, \quad z=\cos \frac{1}{2} \phi,
$$

then

$$
\int_{0}^{\pi} \frac{d \theta}{\sqrt{\left\{1-(m \cos \theta+n)^{2}\right\}}}=\int_{0}^{\pi} \frac{d \phi}{\sqrt{\left(1+m^{2}-n^{2}-2 m \cos \phi\right)}}
$$

or if

$$
m=\frac{r}{a}, \quad n=-\frac{i z}{a}
$$

then finally

$$
\int_{0}^{\pi} \frac{d \theta}{\sqrt{\left\{a^{2}+(z+i r \cos \theta)^{2}\right\}}}=\int_{0}^{\pi} \frac{d \phi}{\sqrt{\left(a^{2}+r^{2}+z^{2}-2 a r \cos \phi\right)}}
$$

the formula in question. 